\title{
PHYSICAL CHARACTERS OF HD 93044 *
}

\author{
LI ZHIPING \\ Beijing Astronomical Observatory, Chinese Academy of Sciences, Beijing 100080, China
}

\begin{abstract}
The measurements of $u v b y H_{\beta}$ of $\mathrm{HD} 93044$ were obtained in April 1991, and the observational results that the star locates nearly in the middle of $\delta$ Scuti instability strip with somewhat deviation to red edge. According to Crawford (1979) and Philip's (1979) calibrations, the effective temperature, absolute visual magnitude and surface gravity are obtained to be $T_{\text {eff }}=7300 \pm 200 \mathrm{~K}, M_{v}=1 .{ }^{\mathrm{m}} 33 \pm 0.39$ and $\log g=3.7 \pm 0.15$, respectively. The observational results of $\Delta m_{1}=0.01$ give an estimate of $[\mathrm{Fe} / \mathrm{H}]=-0.003 \pm 0.18$, so the opinion of metallic deficient is not supported obviously. The observations show the reddening index $E(b-y)$ to be 0.014 which is 1.4 times as large as the standard deviation of Crawford's (1979) statistics.
\end{abstract}

\section{Introduction}

HD 93044 was discovered to be a $\delta$ Scuti variable (Heynderickx 1990, Li et al. 1990.) with a dominant frequency 11.90808 cycle per day (Heynderickx 1990). There is some argument about if it has multiperiods. By means of period analysis method of PDM-technique, Heynderickx (1990) analyzed 214 measurements obtained between December 1984 and February 1987 and pointed out, "one frequency is clearly present and after prewhitening no other significant frequency could be found." But because the fitting is rather poor during some nights, he then explained that it might be that the pulsation amplitude of HD 93044 is variable in some erratic way. On the other hand, by using MEMPOW method $\mathrm{Li}$ et al. (1991) analyzed $177 \mathrm{~V}$ band measurements obtained from April 9 to May 6,1990 and presented two pulsating frequencies of HD 93044, the ratio between the frequencies is about 0.51 which is in agreement with the ratio of radial pulsation fundamental frequency and third overtone one.

Other interesting questions of HD 93044 originate from the metallic abundance and luminosity. From the Geneva photometry measurements, Heynderikx (1990) concluded HD 93044 to be a near zero main sequence and somewhat metallic deficient $\lambda$ Bootis star. On the other hand, by using 72 inch telescope of Lowell Observatory and a $40 \AA / \mathrm{mm}$ spectrograph, Slettbak (1968) obtained a spectral observation and concluded that the $\mathrm{MK}$ classification of HD 93044 is A7 III. By checking the uvby measurements of Slettbak (1968) and the character of pulsating frequencies of HD 93044, $\mathrm{Li}$ et al. (1991) thought a significant reddening index possibly existent. All those opinions above are not in agreement completely. In order to solve these problems, we made a uvby $H_{\beta}$ observations, here we give some results.

- Project supported by Young Foundation of Beijing Astronomical Observatory, China 


\section{Observations and Analysis}

On the night of April 4, 1991, by using the $60 \mathrm{~cm}$ reflector working on DC mode (Shi et al. 1987) and controlled by a microcomputer at Xinglong Station of Beijing Observatory, we observed HD 930.44 in uvby $H_{\beta}$ filters for about two hours. The standard stars were HR 3974 and HR 3951 which were selected from the Catalog of bright uvby $H_{3}$ standard stars (Perry et al. 1987). The observed results are listed in Table I, where $n$ represents the number of observations and $\sigma$ is the standard deviation of observations.

TABLE I

The uvby 3 observations of HD 93044

\begin{tabular}{lllllll}
\hline St.ar & $V$ & $b-y$ & $m_{1}$ & $c_{1}$ & $\beta$ & $n$ \\
\hline HD 93044 & 7.11 & 0.174 & 0.172 & 0.841 & 2.740 & 5 \\
HR 3974 & 4.486 & 0.106 & 0.201 & 0.876 & 2.837 & 5 \\
HR 3931 & 5.35 & 0.416 & 0.234 & 0.388 & 2.599 & 5 \\
$\sigma$ & 0.004 & 0.011 & 0.010 & 0.028 & 0.0095 & \\
\hline
\end{tabular}

According to Crawford's (1979) empirical calibrations of the $u v b y H_{\beta}$ systems, we obtain $(b-y)_{0}=0.188, \delta m_{1}=0.01, \delta c_{1}=0.181$ and $M_{v}=$ $1^{\mathrm{m}} .33 \pm 0.39$, the error of absolute visual magnitude is caused by observations and the uncertainty of the calibrations. Then we obtain the reddening index $E(b-y)=0.014$ and which is 1.4 times as large as the standard deviation of the statistics of the calibrations of Crawford (1979).

Using the empirical formula of Hack (1978) $[\mathrm{Fe} / \mathrm{H}]=-11.3 \Delta m_{1}(b-y) \pm$ 0.18 , we get $[\mathrm{Fe} / \mathrm{H}]=-0.003 \pm 0.18$, and using the grid for the calculation

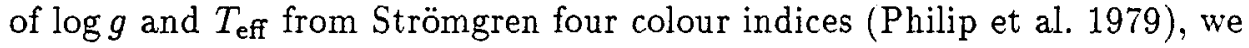
obtain $\log g$ and $T_{\text {eff }}$ to be $3.7 \pm 0.15$ and $7300 \pm 200 \mathrm{~K}$ respectively.

\section{Discussion}

Heyderickx (1990) thought, HD 93044 is rather close to the ZAMS with visual absolute magnitude $1^{\mathrm{m}} .96$. This magnitude is about $0^{\mathrm{m}} .5$ fainter than that we obtained. And according to the MK spectral classification of Slettbak (1968) HD 93044 is about $1^{\mathrm{m}} .0$ above the ZAMS. So we tend to think that the magnitude value of Heynderichx (1990) is larger than the real value and it was caused by neglecting the reddening. Meylan (1980) give the intrinsic temperature parameter $\left(B_{2}-V_{1}\right)$ of different $\mathrm{MK}$ classification, corresponding to A7 III, the value of $\left(B_{2}-V_{1}\right)$ is -0.001 , this is far smaller than the observed value 0.078 of HD 93044 (Heynderickx 1990), this difference implied a significant reddening possibly existent also.

The $\lambda$ bootis type metallic deficient of HD 93044 is not confirmed in our 
observations of $u v b y H_{3}$. Is the metallic abundance index $m_{1}$ not sensitive to the $\lambda$ bootis type star? Or is another cause existent? In order to solve this question, more photoelectric photometry and spectroscopic observations are more useful.

\section{Acknowledgements}

The financial support for author to attend this conference was given by the L.O.C. of I.A.U. Colloquium No. 134. I would like to thank Prof. Kodaira, K., Takeuti, M., Hamada, T., Tanaka, Y., Ando, H., and Kogure, T. et al. for their warm reception during my visit of Japan.

\section{References}

Crawford, D. L.: 1979, Astronomical Journal 84, 1858.

Hack, B.: 1978, Astronomy and Astrophysics 63, 273.

Heynderickx, D.: 1990, Astronomy and Astrophysics 232, 79.

Li Zhiping, Tang Qingquan, Cao Ming: 1990, IBVS, 3467.

Li Zhiping, Jiang Shiyang, Liu Yanying, Cao Ming: 1991, Astronomy and Astrophysics 245,485 .

Meylan, G., Python, M., Hauck, B.: 1989, Astronomy and Astrophysics 90, 83.

Perry, C. L., Olsen, E. H., Crawford, D. L.: 1987, Publications of the ASP 99, 1184.

Philip, G. A., Relyea, L. J.: 1979, Astronomical Journal 84, 1743.

Shi, C. M., Du, B. T., et al.: 1987, Acta Astrophys. Sin. 7, 230.

Slettebak A., Wright R. R., Graham J. A.: 1968, Astronomical Journal 73, 152. 